\title{
An Improved Immune Genetic Algorithm and Its Application in Com- puter-aided Landscape Design
}

\author{
Wang Yao-Kuan* and Liu Yu-Hong
}

School of Software, Nanyang Institute of Technology, Henan Nanyang 473004, China

\begin{abstract}
This paper proposes an improved immune genetic algorithm, and utilizes it in evaluating the results of computer-aided landscape design. After analyzing the related works and the flow chart of the standard immune genetic algorithm, an improved immune genetic algorithm is designed. The main modifications of our proposed immune genetic algorithm lie in the following aspects. 1) We modified the standard immune genetic algorithm using symbolic coding and full binary tree in the chromosomes to describe solutions. 2) The crossover operator with single point is used, and the cross point can be selected from the intermediate nodes and the root nodes. 3) The mutation operator is modified to avoid the dependence of mutation probability on the initial value. 4) The modified immune genetic algorithm not only can keep random global search ability, but also can avoid local premature convergence. Next, the landscape design evaluation results can be obtained by SVM, the parameters of which can be optimized by the proposed modified immune genetic algorithm. Finally, experiments are conducted on three datasets using an index system which is including 17 indexes. Experimental results demonstrate that the proposed scheme can effectively evaluate the quality of computer-aided landscape design.
\end{abstract}

Keywords: Chromosome, crossover, computer-aided design, immune genetic algorithm, landscape design, mutation.

\section{INTRODUCTION}

In the different kinds of feature selection algorithms, the genetic algorithm is an effective solution. The genetic algorithm is biologically inspired and has many mechanisms mimicking natural evolution. It has a great deal of potential in scientific and engineering optimization or search problems. Furthermore, genetic algorithm is naturally applicable to feature selection since the problem has an exponential search space. The traditional works demonstrated evidence for the superiority of the genetic algorithm compared to representative classical algorithms. In recent years, many research papers are published, which have shown advantages of the genetic algorithm for feature selection. However, although they presented experimental data supporting the superiority of genetic algorithm, other literatures involving comparative studies did not give satisfactory arguments $[1,2]$.

In order to extend the performance of the genetic algorithm, immune genetic algorithm is proposed recently. The aim of introducing immune concepts and algorithms into genetic algorithm is theoretically to utilize the local characteristic information for seeking the ways and means of finding the optimal solution when dealing with difficult problems. During the actual operation, Immune genetic algorithm first specifically analyses the pending problem which is regarded as "antigen", where the basic characteristic information which is regarded as "vaccine" is extracted. Afterwards, the characteristic information is handled and transformed to be a solution and the set of total results from this solution is called "antibody" based on the vaccine. Finally the solution is transformed to be the immune operator by a certain format to execute the operation [3, 4].

As is illustrated in Wikipedia, Computer-aided design refers to the use of computer systems to assist in the creation, modification, analysis, or optimization of a design. Computer-aided design software is used to increase the productivity of the designer, improve the quality of design, improve communications through documentation, and to create a database for manufacturing. Computer-aided design output is often in the form of electronic files for print, machining, or other manufacturing operations.

Furthermore, Computer-aided design is an important industrial art extensively used in many applications, such as automotive, shipbuilding, and aerospace industries, industrial and architectural design, prosthetics, and so on. Computeraided design is also widely used to produce computer animation for special effects in movies, advertising and technical manuals, often called digital content creation. The modern ubiquity and power of computers means that even perfume bottles and shampoo dispensers are designed using techniques unheard of by engineers of the 1960s. Because of its enormous economic importance, Computer-aided design has been a major driving force for research in computational geometry, computer graphics and discrete differential geometry $[5,6]$.

As is well known that design is a complex engineering activity, in which computers are more and more involved. The task of design can often be seen as an optimization prob- 
lem in which the parameters or the structure describing the best quality design are sought. The Immune genetic algorithm is made up of a class of search algorithms especially suited to solving complex optimization problems. In addition to parameter optimization, immune genetic algorithms are also suggested for solving problems in creative design, such as combining components in a novel, creative mode. Immune genetic algorithms utilize the notions of evolution in nature to computers and then imitate natural evolution. Particularly, this algorithm finds solutions to a problem by maintaining a population of possible solutions according to the 'survival of the fittest' principle [7].

Based on the above analysis, we present an improved immune genetic algorithm and then use the proposed algorithm in computer-aided design.

The rest of the paper is organized as the following sections. Section 2 introduces the related works. Section 3 illustrates the improved immune genetic algorithm and its application in computer-aided landscape design evaluation. In section 4, a series of experiments are designed and conducted to make performance evaluation. Finally, we conclude the whole paper in section 5 .

\section{RELATED WORKS}

In this section, the related works of this paper are surveyed. Particularly, some representative works are given as follows.

Diabat et al. proposed a multi-echelon reverse logistics network for product returns to minimize the total reverse logistics cost, which consists of renting, inventory carrying, material handling, setup, and shipping costs. Industries need to give more attention to the task of collecting used products from customers and establishing collection facilities. Particularly, a mixed integer non-linear programming (MINLP) model is developed to find out the number and location of initial collection points and centralized return centers required for an effective return and collection system, and also the maximum holding time for aggregation of small volumes of returned products into large shipments [8].

Liang et al. proposed a novel system which applies a combination of two methods of artificial immune and genetic algorithm to diagnose the liver disease. The system architecture is based on artificial immune system. The learning procedure of system adopts genetic algorithm to interfere the evolution of antibody population [9].

Li et al. utilized immune genetic algorithm for pose optimization in latent space of human motion. Firstly, the authors perform human motion analysis in the learnt latent space of human motion. As the latent space is low dimensional and contents the prior knowledge of human motion, it makes pose analysis more efficient and accurate. Furthermore, IGA is used for pose optimization. Compared with genetic algorithm and other evolutionary methods, its main advantage is the ability to use the prior knowledge of human motion. The authors proposed a sequential IGA(S-IGA) algorithm for motion tracking by incorporating the temporal continuity information into the traditional IGA [10].

Chen et al. presented a novel intelligent diagnosis model based on wavelet support vector machine (WSVM) and im- mune genetic algorithm (IGA) for gearbox fault diagnosis. Immune genetic algorithm is developed in this study to determine the optimal parameters for WSVM with the highest accuracy and generalization ability. Moreover, the feature vectors for fault diagnosis are obtained from vibration signal that preprocessed by empirical mode decomposition (EMD)[11].

Tao et al. proposed a modified self-adaptive immune genetic algorithm (MSIGA) with two memory bases to improve the searching ability of the algorithm and maintain population diversity. Performance comparisons with other well-known population-based iterative algorithms show that the proposed method converges quickly to the global optimum and overcomes premature problem. This algorithm is applied to optimize a feed forward neural network to measure the content of products in the combustion side reaction of p-xylene oxidation, and satisfactory results are obtained [12].

Arivudainambi et al. presented a new efficient immune genetic algorithm (IGA) for broadcast scheduling problem in TDMA Ad hoc network. The proposed algorithm improves the transmission utilization by assigning one transmission time slot to one or more non-conflicting nodes such a way that every node transmits at least once in each TDMA frame. An optimum transmission schedule could minimize the length of a TDMA frame while maximizing the total number of transmissions. The aim of this paper is to increase the number of transmissions in fixed Ad hoc network with time division multiple access (TDMA) method, with in a reduced time slot [13].

Liao et al. presented a novel algorithm-Isolation Niche Immune Genetic Algorithm for solving power system BidBased Dynamic Economic Dispatch (INIGA-BDED). Economic Dispatch determines the electrical power to be generated by the committed generating units in a power system so that the generation cost can minimized, while simultaneously satisfying various load demands. Moreover, this paper also proposed the Niche based on the Isolation mechanism which is based on biological possesses. The proposed method effectively ensures diverse group solutions and also has a strong ability to guide evolution. Using the immune genetic algorithm itself is a very good and innovative method for multi-peak model function solutions [14].

Zhou et al. proposed an new immune GA (UIGA) based on the clonal selection strategy and UDS. Based on the bias of samples in the uniform design sampling (UDS) point set, the crossover operation in GA is redesigned. Using the concentrations of antibodies in artificial immune system (AIS), the chromosomes concentration in GA is defined and the clonal selection strategy is designed. In order to solve the maximum clique problem (MCP)[15].

Xue et al. studied on how to optimize enterprise information system (EIS) structure based on time property. Once the EIS structure is formally represented, the time property corresponding to EIS structure expression can be obtained. Thus, aiming at the minimum time property, the EIS structure can be optimized. Afterwards, the EIS structure model is optimized by the improved immune genetic algorithm (IGA) based on binary tree, niche algorithm and self-adaptive operators, and the steps of the improved IGA are presented in detail as well [16]. 
Guo et al. presented a two-stage damage detection method based on Bayesian theory and immune genetic algorithm (IGA) to solve structural multi-damage identification problem. First, structural modal strain energy and frequency are considered as two kinds of information sources, and Bayesian theory is utilized to integrate the two information sources and preliminarily identify structural damage locations. After the damaged locations are determined, immune genetic algorithm is used to identify structural damage extent [17].

Qian et al. designed a novel kinetic model based on the free radical mechanism to simulate the oxidation of $p$-xylene $(\mathrm{PX})$ in a continuous stirred-tank reactor (CSTR) under industrial operating conditions. Correction coefficients are introduced to accurately evaluate the kinetic parameters based on Adaptive Immune Genetic Algorithm (AIGA) due to the significant difference between the nature of PX oxidation conducted in the laboratory SBR and in the industrial CSTR [18].

$\mathrm{Zu}$ et al. proposed a multi-user cognitive radio network resource allocation based on the adaptive niche immune genetic algorithm, and then a fitness function is provided. Simulations are conducted using the adaptive niche immune genetic algorithm, the simulated annealing algorithm, the quantum genetic algorithm and the simple genetic algorithm, respectively [19].

Moghaddam et al. proposed an Immune-based Genetic algorithm (IGA) and a meta-heuristic approach with a new coding scheme to solve MTSP. Furthermore, to overcome the regeneration phenomena in the proposed GA (generating similar chromosomes) which leads to premature convergence, an affinity based approach inspired from Artificial Immune system is employed which results in better exploration in the searching process [20].

\section{THE PROPOSED SCHEME}

Immune genetic algorithm is a global search algorithm based on immune system and the standard genetic algorithm. In this optimization approach, variable parameters are coded into a string known as an antibody (individual). Each of these antibodies has a related fitness value, which can be obtained by the objective function to be maximized or minimized. The immune genetic algorithm is implemented by adjusting the concentration of a population, which is made up different antibodies with different fitness, and then the immune genetic algorithm can efficiently search the new generation with better fitness. Immune genetic algorithm has been tested to have the ability to solve the optimization problem through concentration control and genetic operation applied to antibodies in the population [21-23].

Particularly, immune genetic algorithm is belonged to a kind of improved genetic algorithm based on biology immune mechanism. Particularly, it is one kind of bionic simulated evolutionary algorithm which can imitate and reflect biological immune system, and combined with engineering optimal problems. Based on the original genetic algorithm, immune genetic algorithm could solve the problem of the low searching efficiency, poor individuals variety and premature of genetic algorithm effectively [24], and ensure that algorithm convergence rapidly in globally optimal solution by application of the mechanism of biodiversity maintenance and the mechanism of consistency adjustment of biological immune system [25].

The process of the immune systems construction is shown in Fig. (1). For a given immune system, the antibodies may be a set of thresholds or limitation conditions to the chromosomes, which can be learned from the neighbors by the inference rules. The learning process of antibodies can be calculated as follows.

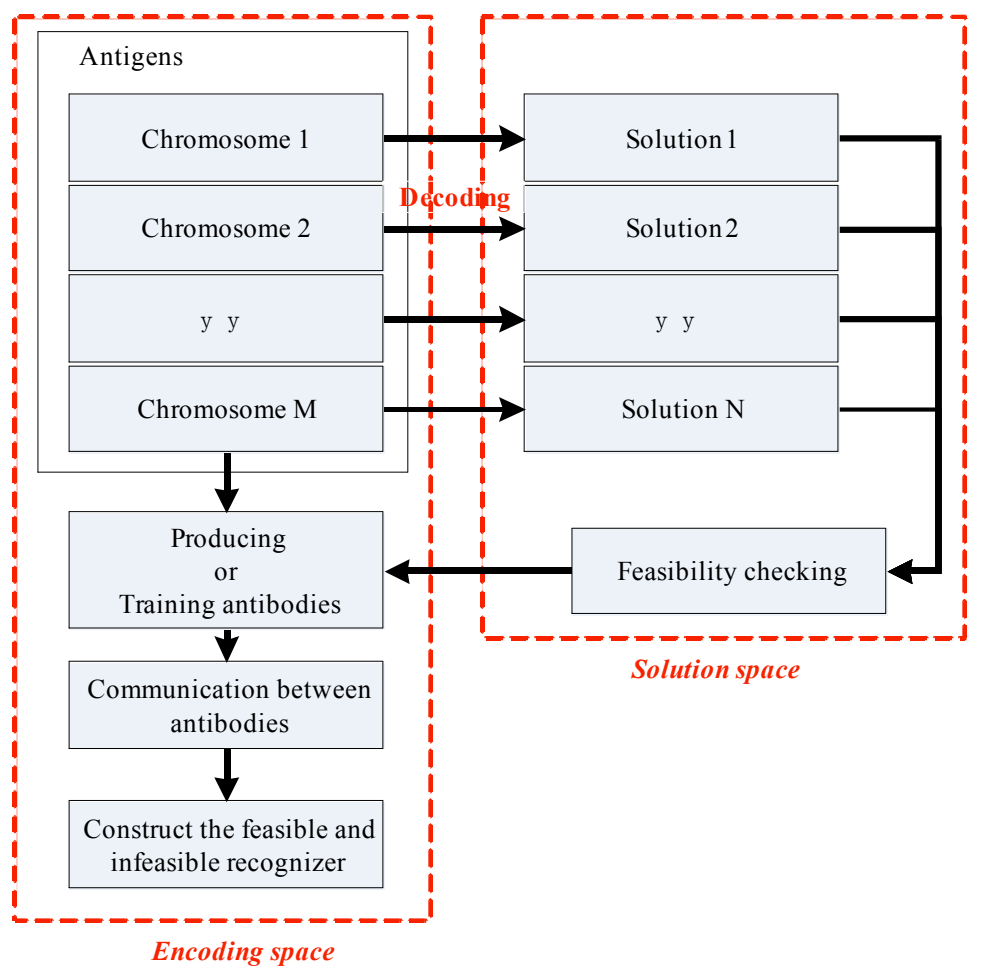

Fig. (1). Process of the immune systems construction. 


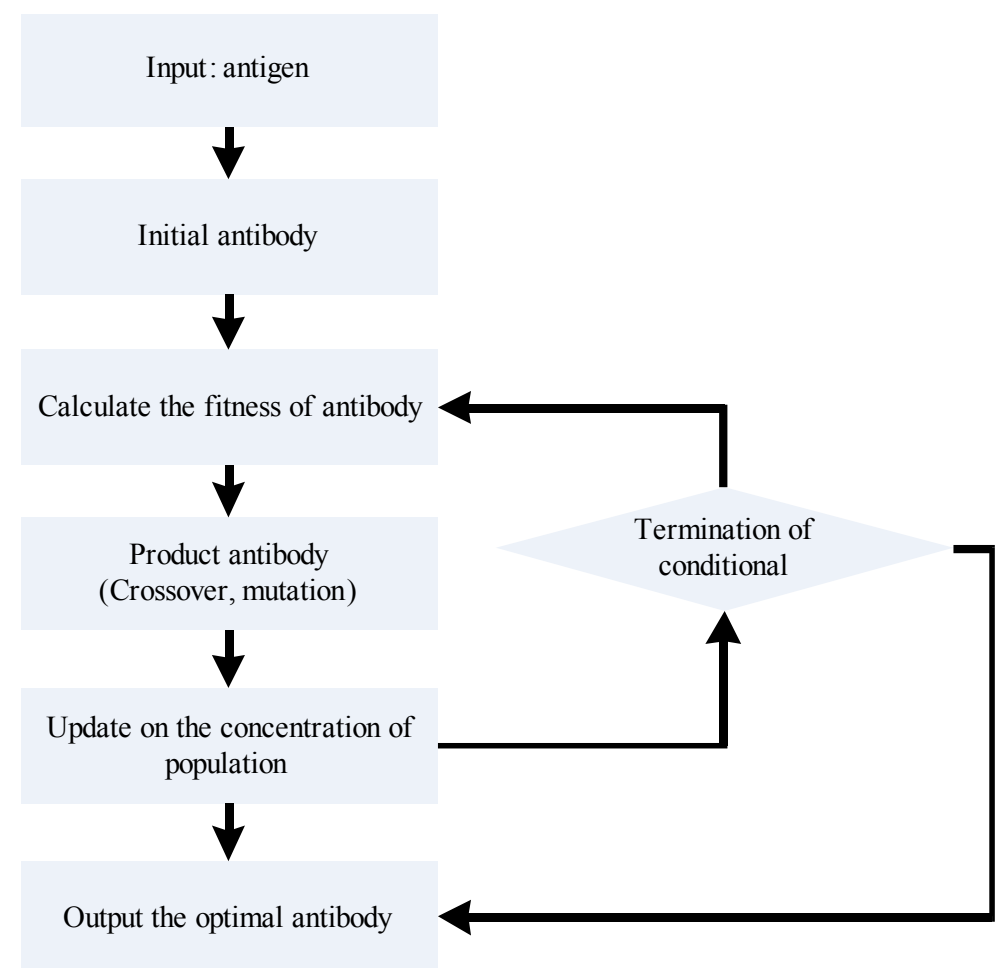

Fig. (2). Flow chart of the standard immune genetic algorithm.

$\operatorname{Th}(i, k)=\operatorname{Th}(i, k-1)+\Delta \operatorname{Rule}\{\operatorname{Th}(i, k-1), \operatorname{Th}(i-1, k)\}, \forall i, \forall k$

where $T h(i, k)$ refers to the threshold of the antibody $i$ after the $k^{\text {th }}$ training, and $i-1$ represents the related antibody which is belonged to its neighbor antibody. $\Delta$ Rule represents the increment based on the rule based learning process.

After the feasible and infeasible recognizer is made, it can be added into the general genetic algorithms to construct a modified immune-genetic algorithm. The process of immune genetic algorithm can be illustrated in Fig. (2). The immune genetic algorithm can be utilized to solve many constrained optimization problems effectively.

In this paper, we modified the standard immune genetic algorithm by utilizing symbolic coding and full binary tree in the chromosomes to describe solutions. Particularly, all the chromosomes' fitness are modified by the following equation.

$\operatorname{sh}(d)=\left\{\begin{array}{c}1-\frac{d}{\sigma_{\text {share }}}, d \leq \sigma_{\text {share }} \\ 0, \text { otherwise }\end{array}\right.$

where $\sigma_{\text {share }}$ refers to the radius of niche and $d$ can be computed by the following equation.

$d=\frac{H(\text { opi }, o p j)}{N \cdot k}$

where $H$ (opi,opj) represents the hamming distance between opi and opj, $N$ denotes the number genes in the chromosome, and $k$ means the number of chromosomes in each sub-group. Afterwards, the sharing fitness function is represented in Eq.4

$\varepsilon\left(\chi_{o p i}\right)=\frac{\varepsilon^{\prime}\left(\chi_{o p i}\right)}{\sum_{o p j=1}^{N} \operatorname{sh}\left(\chi_{o p i}, \chi_{o p j}\right)}$

Particularly, the crossover operator with single point is used in this paper, and the cross point is chosen between the intermediate nodes and the root nodes. Next, we exchange the two nodes chosen in the above process, and then two chromosomes with higher fitness of all the 4 replace the initial two ones.

To avoid the mutation probability's dependence on the initial value, self-adaptive crossover probability is designed as follows.

$p_{c}=\left\{\begin{array}{c}p_{c 1}-\frac{\left(p_{c 1}-p_{c 2}\right) \cdot\left(\delta^{\prime}-\delta_{a v g}\right)}{\delta_{\max }-\delta_{\text {avg }}}, \delta^{\prime} \geq \delta_{\text {avg }} \\ p_{c 1}, \text { otherwise }\end{array}\right.$

where $\delta_{\max }$ refers to the highest fitness in the current population, $\delta_{a v g}$ represents the average fitness in the current population. Particularly, $\delta$ means the higher fitness of the two crossover individuals, and $p_{c 1}$ and $p_{c 2}$ means the initial values which can be computed by the self-adaptive crossover probability.

In the proposed immune genetic algorithm, the mutation operator is modified as well to avoid the dependence of mutation probability on the initial value. The self-adaptive mutation probability is obtained by the following equation. 


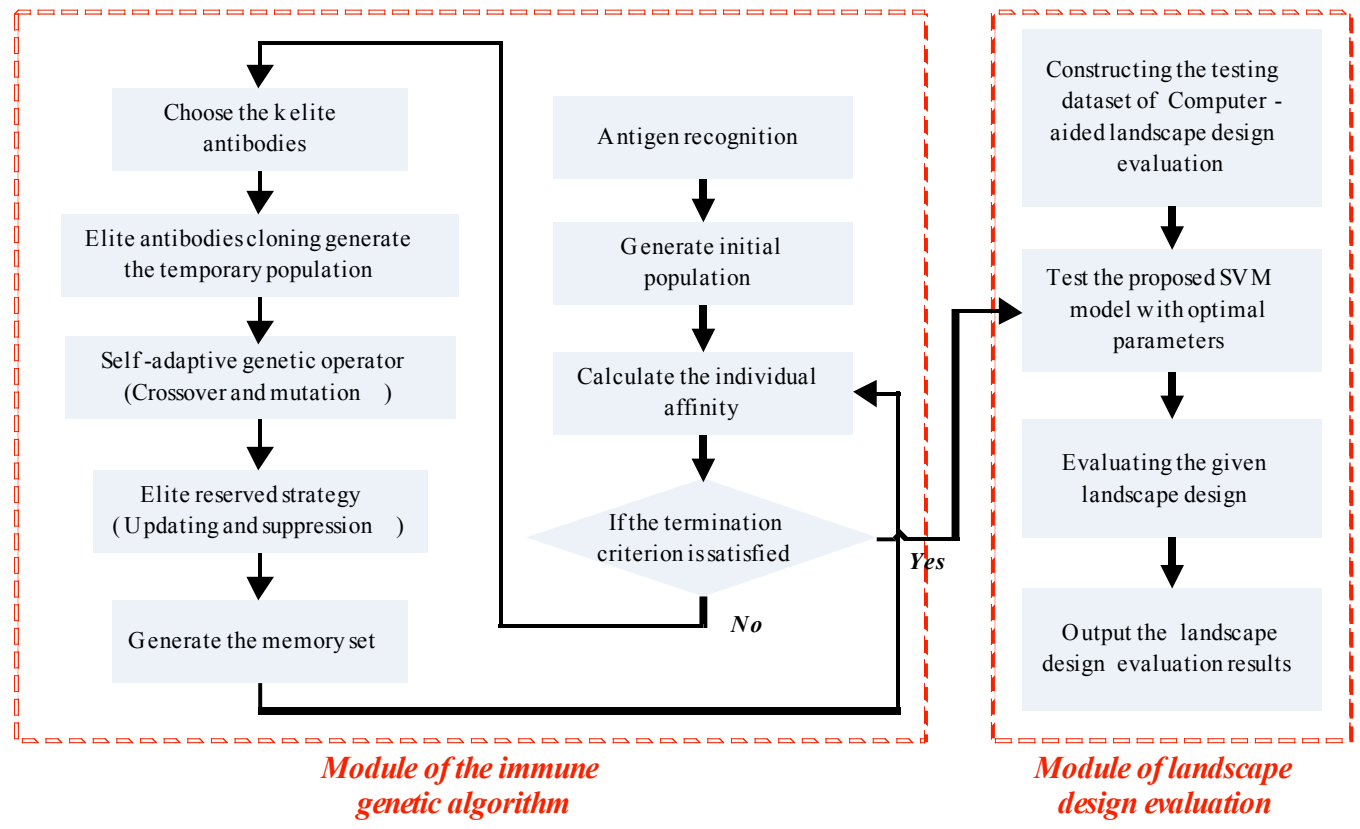

Fig. (3). Process of the proposed improved immune genetic algorithm based computer-aided landscape design evaluation.

$p_{m}=\left\{\begin{array}{c}p_{m 1}-\frac{\left(p_{m 1}-p_{m 2}\right) \cdot\left(\delta_{\text {avg }}-\delta\right)}{\delta_{\max }-\delta_{\text {avg }}}, \delta \geq \delta_{\text {avg }} \\ p_{m 1}, \text { otherwise }\end{array}\right.$

where $\delta_{\max }$ refers to the best fitness in the current population, and $\delta_{\text {avg }}$ means the average fitness in the current population, $\delta$ represents the fitness of the mutation individual. $p_{m 1}$, $p_{m 2}$ refer to the initial values for computing self-adaptive mutation probability respectively.

After the improved immune genetic algorithm is given, the landscape design evaluation results can be obtained by SVM, the parameters of which can be optimized by the proposed immune genetic algorithm. Next, we will illustrate how to optimize the support vector machine with the proposed immune genetic algorithm.

Using the Morlet wavelet kernel function, the optimal decision function of the SVM can be represented as follows.

$$
f(x)=\operatorname{sgn}\left[\begin{array}{l}
\sum_{j=1}^{n} \alpha_{j} \cdot \prod_{i=1}^{d}\left[\cos \left(1.75 \cdot \frac{\left(x_{j}^{i}-x_{j}^{i^{\prime}}\right)}{a_{j}^{i}}\right)\right] \\
\cdot \exp \left[-\frac{\left\|x_{j}^{i}-x_{j}^{i^{\prime}}\right\|^{2}}{2 \cdot a_{j}^{i^{2}}}\right]+b
\end{array}\right]
$$

where $n$ denotes the training sample number, and $x_{j}^{i}$ refers to the $i^{\text {th }}$ variable in the $j^{\text {th }}$ training sample.

Based on the above optimal decision function, immune selection, immune mutation, immune renewal and the dynamic adjustment strategy can be converted to meet the requirement of large scale optimal computation. On the other hand, the immune memory function is adopted in immune genetic algorithm to improve whole search ability and quicken search speed. Hence, immune genetic algorithm not only can keep random global search ability, but also avoid local premature convergence, which is more suitable for parameters optimization.

Afterwards, the results of computer-aided landscape design evaluation can be obtained by the above SVM based on training dataset in Fig. (3).

\section{EXPERIMENTAL RESULTS}

Landscape design refers to an independent profession and a design and art tradition, practised by landscape designers, combining nature and culture. In contemporary practice, landscape design bridges between landscape architecture and garden design. Particularly, landscape design focuses on both the integrated master landscape planning of a property and the specific garden design of landscape elements and plants within it. The practical, aesthetic, horticultural, and environmental sustainability components merit Landscape design inclusion. Landscape designers usually collaborate with related disciplines such as architecture and geography, soils and civil engineering, surveying, landscape contracting, botany, and artisan specialties.

Based on the above illustration of landscape design, how to effectively evaluate the given landscape design is of great importance in the process of landscape design. However, evaluating the landscape design is quite hard. The reasons lie in that when evaluating the landscape design, many factors should be considered, including the aesthetics, practicality, economy, ecology, and so on.

In the following section, we will test the performance of the proposed improved immune genetic algorithm through evaluating landscape design results. Before evaluating the landscape design, the index systems should be determined in advance. The indexes and related weights we used in this experiment are given in Table $\mathbf{1}$ as follows. Particularly, the index weights are obtained by the Analytic hierarchy process algorithm. 
Table 1. The index of landscape design evaluation.

\begin{tabular}{|c|c|c|}
\hline \multirow{3}{*}{ Overall } & I1: Green rate & 0.099 \\
\hline & I3: road coverage & 0.083 \\
\hline & I4: building floor area ratio & 0.073 \\
\hline \multirow[t]{2}{*}{ Practicality } & I6: square number & 0.047 \\
\hline & I7: pavilion number & 0.050 \\
\hline Layout reasonable & I8: Distance between building and wall & 0.044 \\
\hline \multirow{6}{*}{ Appearance } & I12: Plant species & 0.049 \\
\hline & I13: Ground height difference & 0.051 \\
\hline & I14: The number of lake island & 0.051 \\
\hline & I15: The number of landscape axis landscape & 0.053 \\
\hline & I16: Landscape facade height & 0.058 \\
\hline & I17: Types night lighting & 0.048 \\
\hline
\end{tabular}

Table 2. Evaluation level for the given fifty testing samples.

\begin{tabular}{|c|c|}
\hline Testing Sample & Evaluation Level \\
\hline \hline S1 & Good \\
\hline S2 & Ordinary \\
\hline S3 & Good \\
\hline S4 & Ordinary \\
\hline S5 & Ordinary \\
\hline S6 & Excellent \\
\hline S7 & Excellent \\
\hline S8 & Good \\
\hline S9 & Good \\
\hline S10 & Good \\
\hline S11 & Good \\
\hline S12 & Excellent \\
\hline S13 & Good \\
\hline S14 & Excellent \\
\hline S15 & Excellent \\
\hline
\end{tabular}

Afterwards, each of the evaluation parameters are used as input data, landscape design evaluation level is regarded as the output by SVM to seek non-linear relationship between them. The evaluation level of training and testing data is defined as "Excellent", "Good", and "Ordinary" (in Table.2). For each landscape design evaluation dataset, 240 samples are used as training data, and other 240 are utilized as the testing data. Three datasets for landscape design evaluation are utilized in this experiment. Hence, the problem of landscape design evaluation can be converted as the problem of classification. Next, fifty testing data are given in Table.3 as follows, and the values of all indexes are normalized.

Next, in order to make performance comparison, the proposed modified IGA based landscape design evaluation is compared with the methods based on genetic algorithm (GA) and immune genetic algorithm (IGA). The results are listed in Fig. (4).

Based on the above experimental results, in can be seen that the proposed scheme is effective in computer-aided landscape design evaluation. The reasons lie in the following aspects:

(1) Compared with GA, the standard IGA has the following advantages: a) immune memory function, b) maintaining antibody diversity function, c) self-adjustment function. So IGA has its own unique advantages while maintaining global parallel search. 
Table 3. Examples of testing samples.

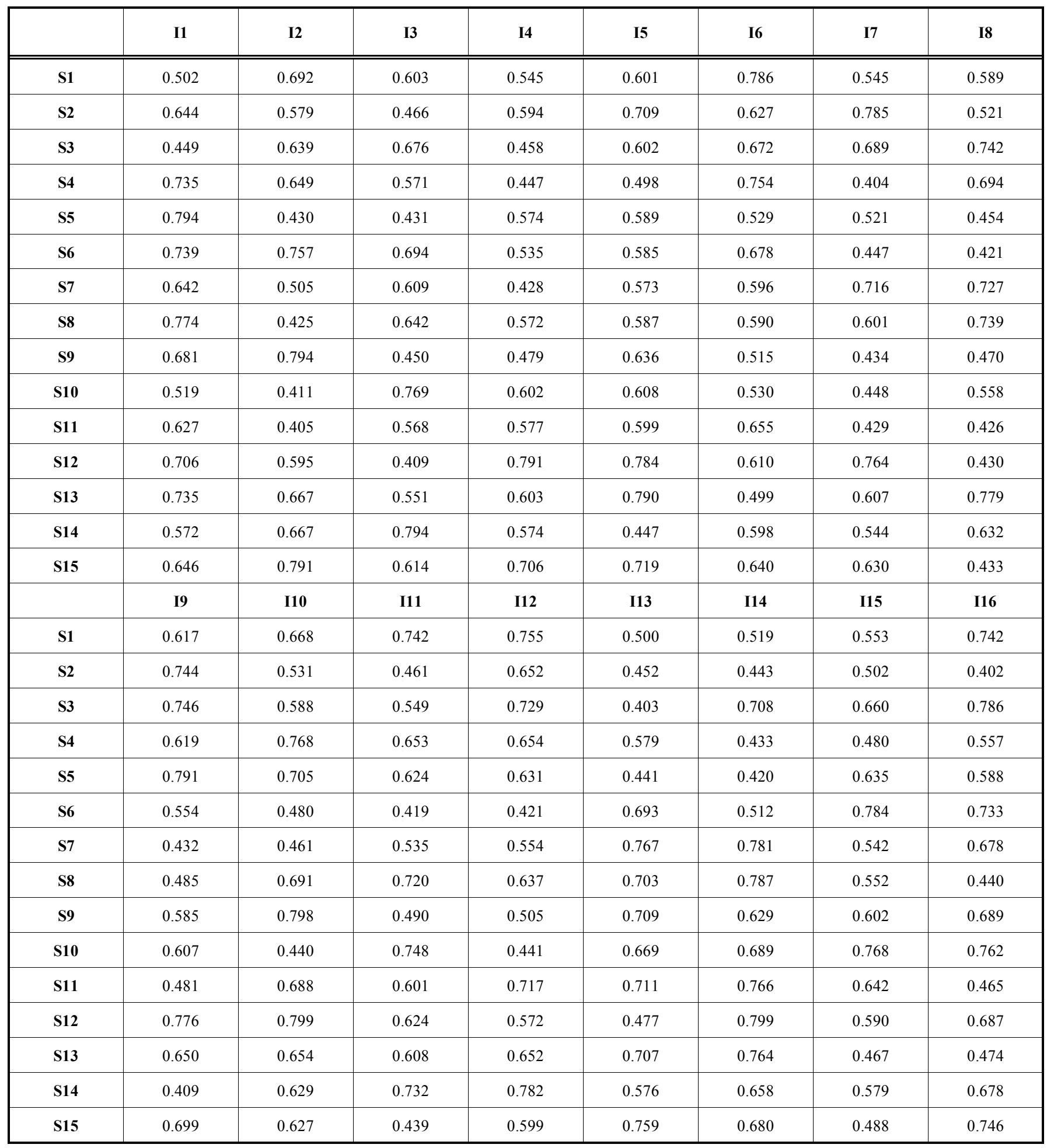

(2) Compared with GA and standard IGA, our modified IGA has the following four advantages:

(a) We modified the standard immune genetic algorithm by utilizing symbolic coding and full binary tree in the chromosomes to describe solutions. Particularly, all the chromosomes' fitness is modified as well. (b) The crossover operator with single point is used in this paper, and the cross point is chosen between the intermediate nodes and the root nodes.

(c) In the proposed immune genetic algorithm, the mutation operator is modified to avoid the dependence of mutation probability on the initial value. 
(d) Our modified immune genetic algorithm not only can keep random global search ability, but also can avoid local premature convergence, which is more suitable for parameters optimization.

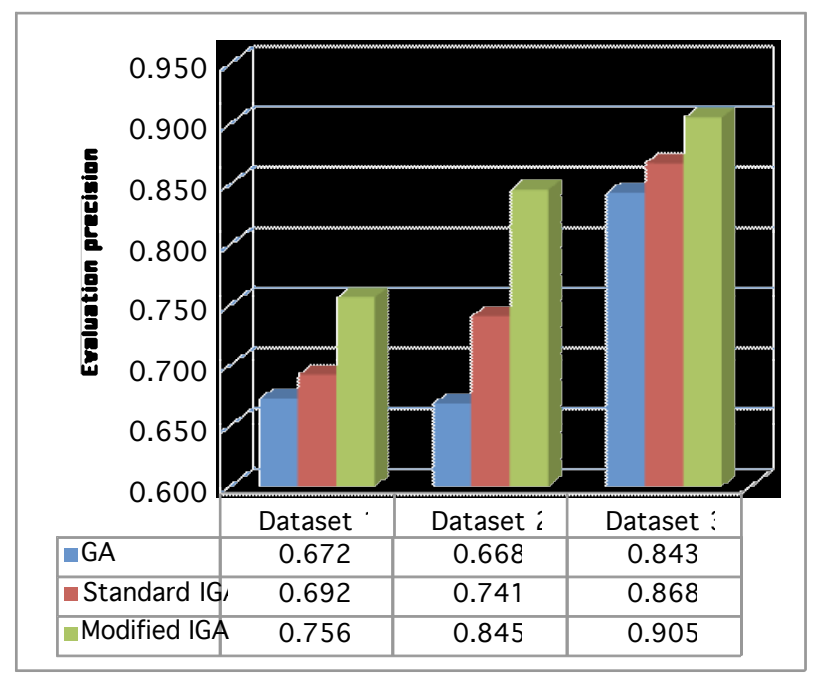

Fig. (4). Performance evaluation for different methods on the three datasets.

\section{CONCLUSION}

In this paper, we present an improved immune genetic algorithm, and then use this algorithm in evaluating the results of computer-aided landscape design. Firstly, we modified the standard immune genetic algorithm using symbolic coding and full binary tree in the chromosomes to describe solutions. Secondly, the crossover operator with single point is used, and the cross point can be selected from the intermediate nodes and the root nodes. Thirdly, a new mutation operator is designed to avoid the dependence of mutation probability on the initial value. Fourthly, the landscape design evaluation results are obtained by SVM. Particularly, the parameters of SVM classifier are optimized by the proposed modified immune genetic algorithm.

\section{CONFLICT OF INTEREST}

The authors confirm that this article content has no conflict of interest.

\section{ACKNOWLEDGEMENTS}

Declared none.

\section{REFERENCES}

I. S. Oh, J.S. Lee, and B. R. Moon, "Hybrid genetic algorithms for feature selection", IEEE Transactions on Pattern Analysis and Machine Intelligence, vol.26, no.11, pp.1424-1437, 2004.

[2] C.Y. Liu, "An improved adaptive genetic algorithm for the multidepot vehicle routing problem with time window", Journal of Networks, vol.8, no.5, pp.1035-1042, 2013.

[3] X.D. Xu, and C.X. Li, "Research on immune genetic algorithm for solving the job-shop scheduling problem", International Journal of Advanced Manufacturing Technology,vol. 34, no.7-8, pp.783-789, 2007.

[4] Y.S. Ding, and T.L. Zhang, "Using Chou's pseudo amino acid composition to predict subcellular localization of apoptosis proteins: An approach with immune genetic algorithm-based ensemble classifier", Pattern Recognition Letters, vol.29, no.13, pp.1887-1892, 2008

[5] J. A. McCammon, R. E. Amaro, and R. Baron, "An improved relaxed complex scheme for receptor flexibility in computer-aided drug design", Journal of Computer-aided Molecular Design, vol.22, no. 9, pp.693-705, 2008.

[6] R. Custelcean, J. Bosano, P. Bonnesen, "Computer-Aided Design of a Sulfate-Encapsulating Receptor", Angewandte Chemieinternational Edition, vol.48, no.22, pp.4025-4029, 2009.

[7] G. Renner, A. Ekart, "Genetic algorithms in computer aided design”, Computer-aided Design, vol.35, no.8, pp.709-726, 2003.

[8] A. Diabat, D. Kannan, and M. Kaliyan, "An optimization model for product returns using genetic algorithms and artificial immune system", Resources Conservation And Recycling, vol.7,no.4, pp.156-169, 2013.

[9] C. Liang, and L. Peng, "An Automated Diagnosis System of Liver Disease using Artificial Immune and Genetic Algorithms", Journal of Medical Systems, vol.37, no.2, Article No. 9932, 2013.

[10] Y. Li, and Z. Sun, "Articulated Human Motion Tracking Using Sequential Immune Genetic Algorithm", Mathematical Problems in Engineering, Article NO. 921510, 2013.

[11] F. Chen, B. Tang, and R. Chen, "A novel fault diagnosis model for gearbox based on wavelet support vector machine with immune genetic algorithm", MEASUREMENT, vol.46, no.1, pp.220-232, 2013.

[12] L. Tao, X. Kong, and W. Zhong, "Modified Self-adaptive Immune Genetic Algorithm for Optimization of Combustion Side Reaction of p-Xylene Oxidation", Chinese Journal of Chemical Engineering, vol.20, no.6, pp.1047-1052, 2012.

[13] D. Arivudainambi, and D. Rekha, "Broadcast Scheduling Problem in TDMA Ad Hoc Networks using Immune Genetic Algorithm", International Journal of Computers Communications \& Control, vol.8, no.1, pp.18-29, 2013.

[14] G. C. Liao, "Integrated Isolation Niche and Immune Genetic Algorithm for solving Bid-Based Dynamic Economic Dispatch", International Journal of Electrical Power \& Energy Systems, vol.42, no.1, 264-275, 2012.

[15] B. D. Zhou, H. L. Yao, and M. H. Shi, “An new immune genetic algorithm based on uniform design sampling", Knowledge and Information Systems, vol.31, no.2, pp.389-403, 2012.

[16] C. Xue, L. Dong, and J. Liu, "Enterprise information system structure optimization based on time property with improved immune genetic algorithm and binary tree", Computers \& Mathematics with Applications, vol.63, no.7, pp.1155-1168, 2012.

[17] H. Y. Guo, and Z. L. Li, "Structural damage identification based on Bayesian theory and improved immune genetic algorithm", Expert Systems with Applications, vol.39,no.7, pp.6426-6434, 2012.

[18] F. Qian, L. Tao, and W. Sun, "Development of a Free Radical Kinetic Model for Industrial Oxidation of p-Xylene Based on Artificial Neural Network and Adaptive Immune Genetic Algorithm", ustrial \& Engineering Chemistry Research,vol.51, no.8, pp.3229-3237, 2012.

[19] Y.X. Zu, and J. Zhou, "Multi-user cognitive radio network resource allocation based on the adaptive niche immune genetic algorithm", Chinese Physics B, vol.21, no.1, Article No. 019501, 2012.

[20] M. E. Moghaddam, M. R. Bonyadi, "An Immune-based Genetic Algorithm with Reduced Search Space Coding for Multiprocessor Task Scheduling Problem", International Journal of Parallel Programming, vol.40, no.2, pp. 225-257, 2012.

[21] T. Chen, and Y. Hsieh, "Using immune-based genetic algorithms for single trader's periodic marketing problem", Mathematical and Computer Modelling, vol.48, no.3, pp.420-428, 2012.

[22] Z. Su, P. Wang, and X. Yu, "Immune genetic algorithm based adaptive evidential model fro estimating unmeasured parameter: Estimating levels of coal powder filling in ball mill", Expert Systems with Applications, vol.37, no.7, pp.5246-5258, 2010.

[23] D. Wang, R. Y. K. Fung, and W. H. Ip, "An immune-genetic algorithm for introduction planning of new products", Computers \& Industrial Engineering, vol.56, no.3, pp.902-917, 2009. 
[24] C. Ni, J. Ding, and Z. Li, "Study on immune algorithm based on superior antibodies and its convergence property", Systems Engineering, vol.20, no.3, pp.72-75, 2002.
[25] J. S. Chun, M. K. Kim, and H. K. Jung, "Shape optimization ofelectronic devices using immune algorithm", IEEE Trans. On Magnetics, vol.33, no.2, pp.1876-1879, 1997.

Received: September 16, 2014

Revised: December 23, 2014

Accepted: December 31, 2014

(C) Yao-Kuan and Yu-Hong; Licensee Bentham Open.

This is an open access article licensed under the terms of the Creative Commons Attribution Non-Commercial License (http://creativecommons.org/licenses/by-nc/3.0/) which permits unrestricted, non-commercial use, distribution and reproduction in any medium, provided the work is properly cited. 